\title{
Effect of Compliant Interface on the Curvature and Stress of Micron-Size Film-Substrate Composite Structure*
}

\author{
Yun Liu \\ Faculty of Information and Automation \\ Kunming University of Science and Technology \\ Kunming, Yunnan Province 650051, China
}

\author{
Yin Zhang \\ State Key Laboratory of Nonlinear Mechanics (LNM) \\ Institute of Mechanics, Chinese Academy of Sciences \\ Beisihuangxilu 15, Beijing, 100080, China \\ zhangyin@Inm.imech.ac.cn
}

\begin{abstract}
The stress inside the film or substrate can be evaluated by the Stoney's formula and its modified/extended formula, which links the curvature to the stress state inside the composite. However, the Stoney's formula and its modified/extended formula assume the rigid interface, which allows no interfacial slip. The compliance of interface results in an interfacial slip, which can significantly affect the distribution of stress inside the film and substrate. The interface layer with the effect of interfacial slip plays the vital role of stress transfer between the film and substrate. The curvatures of film and substrate are shown to be different in general with the presence of interfacial slip and deviate significantly from that predicated by the models of rigid interface. This could be a serious error source when using the models of rigid interface to evaluate the stress inside the film or substrate, especially when the dimension of the composite is the order of several microns. A model which extends the application of Stoney's formula to the case of composite with interfacial slip is presented.
\end{abstract}

Index Term - Film-substrate composite, interface, microsystem, size effects

\section{INTRODUCTION}

The rigid interface model (also called ideal interface [1, 2], perfectly bonded interface [3] or coherent interface [4]), which allows no interfacial slip is a strong constraint condition because the strains inside two solid phases separated by an interface can be independent [4]. During the fabrication and processing of film-substrate layered structures, such as chemical vapour deposition, wafer bonding, sputtering, doping/diffusion, implantation, thermal annealing process, heteroepitaxial film growth etc., defects like dislocation, twin, cavities etc appear. Therefore, the interface may not be composed of $100 \%$ well-fused bonds. The formation of amorphous layer and dangling bonds in some regions between the two phases also result in the weakly bonded interface areas. All these above will reduce the overall interface adhesion for sure [5]. Both experiment and theoretical analysis show that the analysis of rigid interface model errors more and more when the size of film-substrate composite shrinks in micron order $[1,2]$.

Almost all solid-state electronic components have the basic structure of a substrate as platform supporting various thin film structures [6]. Stress is of a great concern for the reliability of those composite structures [6,7]. As the material properties of film and substrate such as Young's modulus, lattice parameters, coefficients of thermal expansion may be different and also due to the stress build-up during fabrication and processing, the stress inside the film and substrate can be different and result in the deflection of composite structure to relax stress [8]. The following Stoney's 1909 formula [9] serves the cornerstone of relating the stress/force inside film to the curvature of a composite structure

$$
\kappa_{S t}=\frac{6 f}{h_{s}^{2} E_{s}} .
$$

$\kappa_{S t}$ is the curvature and $f$ is the force per unit length inside film (when the film is very thin, $f$ is the surface stress [10]). $h_{s}$ is the substrate thickness and $E_{s}$ is the substrate effective Young's modulus. The applicability of above Stoney formula relies on several assumptions, which are well summarized by Freund et al. [11] Many models are developed to relax one or some of the assumptions to extend Stoney formula to a more generalized and realistic application, for example, by considering the effects of thin substrate thickness [11, 12, 13], large deformation [11], nonisotropic stress [14], temperature gradient [15], stress gradient [12, 16], residual axial force, boundary conditions, length [10, 17], diffusion effect [18], plastic deformation [19]. However, all the analyses above deal with the scenario of ideal interface, i.e. no interfacial slip. During their derivation, the condition of no interfacial slip is either explicitly enforced by the compatibility/continuity condition at the interface $[13,15]$ or implicitly used by assuming one single strain/displacement variable for both film and substrate layers $[10,11,12,14,16,17,18,19]$. Here a

\footnotetext{
* This work is supported by both the National Natural Science Foundation of China (NSFC, Grant No.10502050) and the Scientific Research Foundation for the Returned Overseas Chinese Scholars, State Education Ministry of China.
} 
model incorporating effects of both the interfacial slip and composite dimensions is presented and its results are compared with those predicted by the ideal interface model. When there is no interfacial slip, the film and substrate share a common curvature $[11-13,15-18]$. With the interfacial slip, the analysis presented in this paper shows that the film and substrate do not necessarily share a common curvature. The curvatures of the film and substrate can be significantly different depending on the interfacial properties. Also the curvatures of the film and substrate with interfacial slip can deviate dramatically from that with an ideal interface.

\section{MODEL DEVELOPMENT}

\section{A. Strains inside Film and Substrate due to the Lattice Mismatch}

This paper analyses the curvatures of the film/substrate of heteroepitaxial growth due to lattice mismatch as the case study. The model development section consists of three parts. The first part is to derive the strain distribution inside the film and substrate due to lattice mismatch and without deflection. With the strain distribution, the film/substrate composite has to deflect to accommodate the bending moment. The second and third parts are to derive such deflection with and without the interfacial slip.

The lattice mismatch induced strains inside the film and substrate have the following relations [12]

$$
\varepsilon_{f}-\varepsilon_{s}=\varepsilon_{m}, \quad E_{1} t_{1} \varepsilon_{f}+E_{2} t_{2} \varepsilon_{s}=0 .
$$

$\varepsilon_{f}$ and $\varepsilon_{s}$ are the strains induced by lattice mismatch in the film and substrate, respectively. $\varepsilon_{m}$ is the mismatch strain defined as $\varepsilon_{m}=\left(a_{s}-a_{f}\right) / a_{f}[12]\left(a_{f}\right.$ and $a_{s}$ are the lattice parameters of the film and substrate, respectively). $E_{1}$ and $E_{2}$ are the effective Young's moduli of the film and substrate. $t_{1}$ and $t_{2}$ as shown in Fig. 1(a) are the thickness of the film and substrate. The first equation in (2) is the compatibility condition and the second one is the Newton' $\mathrm{s}$ 3rd law. From (2), $\varepsilon_{f}$ and $\varepsilon_{s}$ are solved as follows

$\varepsilon_{f}=\varepsilon_{m} \frac{E_{2} t_{2}}{E_{1} t_{1}+E_{2} t_{2}}, \quad \varepsilon_{s}=-\varepsilon_{m} \frac{E_{1} t_{1}}{E_{1} t_{1}+E_{2} t_{2}}$.

Clearly, $\mathcal{E}_{f}$ and $\mathcal{E}_{s}$ have the opposite signs, which implies that there is a bending moment in the film/substrate bilayer and the bilayer must deflect. The following derivation is about how the bilayer accommodates this bending moment by deflecting with and without interfacial slip. (a) Two coordinate sets at the mid-planes of two layers

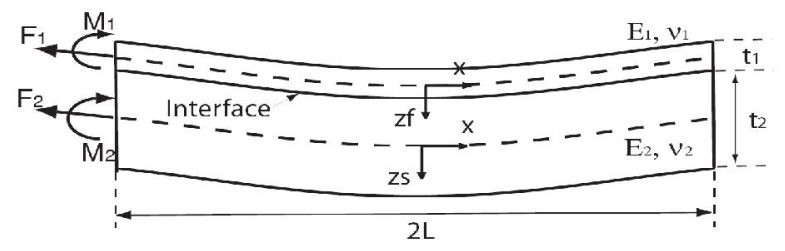

(b) Illustration of interfacial normal and shear stresses of S-L model

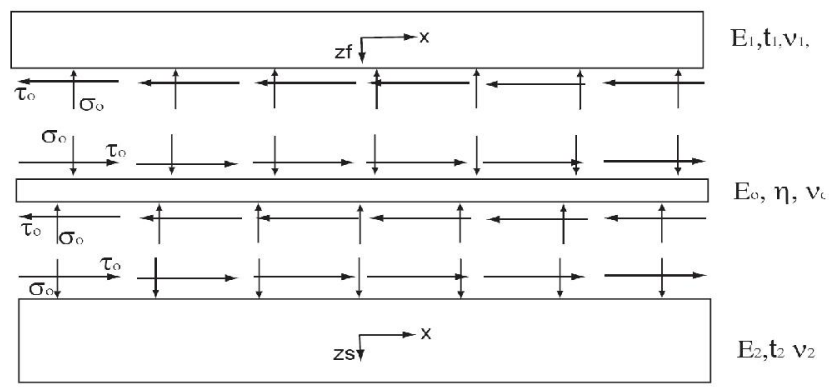

Fig.1. Two coordinate systems at the mid-planes of two layers. (b): Illustration of interfacial normal stress $\left(\sigma_{o}\right)$ and shear stress $\left(\tau_{o}\right)$ in the S-L model.

\section{B. Timoshenko Model of Rigid Interface}

The equilibrium requires the balance of both force and moment, which gives the following two equations

$$
F_{1}+F_{2}=0 \text {, }
$$

And

$$
M_{1}+M_{2}-F_{1} \frac{t_{1}}{2}+F_{2} \frac{t_{2}}{2}=0 .
$$

$F_{1}$ and $M_{1}$ are the force and moment per unit width acting inside the film as shown in Fig. 1(a). $F_{2}$ and $M_{2}$ are those inside the substrate. From (4), it gives the following equation

$$
F_{1}=P(x)=-F_{2} \text {. }
$$

Substitute (6) into (5), it gives

$$
\frac{P(x)\left(t_{1}+t_{2}\right)}{2}=M_{1}+M_{2} .
$$

The longitudinal normal strains of the two layers are expressed as the following

$$
\begin{aligned}
& \frac{d u_{1}(x, z)}{d x}=\mathcal{E}_{f}+\frac{P(x)}{E_{1} t_{1}}+\frac{z}{\rho}, \quad-\frac{t_{1}}{2} \leq z \leq \frac{t_{1}}{2}, \\
& \frac{d u_{2}(x, z)}{d x}=\varepsilon_{s}-\frac{P(x)}{E_{2} t_{2}}+\frac{z}{\rho}, \quad-\frac{t_{2}}{2} \leq z \leq \frac{t_{2}}{2} .
\end{aligned}
$$

$u_{1}(x, z)$ and $u_{2}(x, z)$ are the longitudinal displacements of the film and substrate. As shown in Fig. 1(a), two sets of coordinates are used in Timoshenko's model. $z_{f}$ and $z_{s}$ start 
from the mid-planes of the film and substrate, respectively.

$\mathrm{P}(\mathrm{x})$ is the constraint force per unit width. $\rho$ is the radius of curvature. Because curvature $\kappa=1 / \rho,(8)$ assumes that the film and substrate share a common curvature. The bending moment $\left(M_{i}, \mathrm{i}=1,2\right)$ has the following relation with $\rho$ :

$$
M_{i}=\frac{E_{i} t_{i}^{3}}{12 \rho} .
$$

Substitute (9) into (7), the following equation is obtained

$$
\frac{1}{\rho}=\frac{6 P(x)\left(t_{1}+t_{2}\right)}{E_{1} t_{1}^{3}+E_{2} t_{2}^{3}} .
$$

The compatibility condition at the interface requires that

$$
\mathcal{E}_{f}+\frac{P(x)}{E_{1} t_{1}}+\frac{t_{1}}{2 \rho}=\mathcal{E}_{s}-\frac{P(x)}{E_{2} t_{2}}-\frac{t_{2}}{2 \rho}
$$

(11) physically indicates the continuity of strain at the interface, i.e. that there is no slip between the layers. From (11), $P(x)$ is solved as a constant as follows

$$
P(x)=\frac{-\varepsilon_{m}}{1 /\left(E_{1} t_{1}\right)+1 /\left(E_{2} t_{2}\right)+3\left(t_{1}+t_{2}\right)^{2} /\left(E_{1} t_{1}^{3}+E_{2} t_{2}^{3}\right)} .
$$

Substitute this $\mathrm{P}(\mathrm{x})$ in (12) into (10) and the curvature $\left(\kappa_{T}\right)$ is solved also as a constant as the following

$$
\kappa_{T}=\frac{1}{\rho}=\frac{\kappa_{S t}\left(1+\frac{t_{1}}{t_{2}}\right)}{1+4 \frac{t_{1}}{t_{2}} \frac{E_{1}}{E_{2}}+6 \frac{t_{1}^{2}}{t_{2}^{2}} \frac{E_{1}}{E_{2}}+4 \frac{t_{1}^{3}}{t_{2}^{3}} \frac{E_{1}}{E_{2}}+\frac{t_{1}^{4}}{t_{2}^{4}} \frac{E_{1}^{2}}{E_{2}^{2}}}
$$

$\kappa_{S t}$ is the Stoney formula in (1) when $f$ is set as

$f=-\varepsilon_{m} E_{1} t_{1}$. The Timoshenko model is a free body

diagram analysis with the introduction of constraint force $\mathrm{P}(\mathrm{x})$. The curvature of (13) is exactly the same as that derived by Freund and Suresh [12] who use an energy approach.

\section{S-L Model of Non-ideal Interface}

The S-L model $[2,8]$ is adopted here to study the interfacial slip effect. In the S-L model, an interfacial layer is assumed. The interfacial normal stress $\left(\sigma_{o}(x)\right)$ and shear stress $\left.\left(\tau_{o}(x)\right)\right)$ due to interfacial slip is illustrated in Fig. 1(b). Here the detailed derivation for this particular problem can not be given due to the limitation of presentation space. But its derivation is similar to the very detailed derivation presented by Murray and Noyan for the problem induced by the thermal expansion [2]. The two governing equations for the interfacial stresses are as follows

$$
\begin{aligned}
& \frac{d^{6} \sigma_{o}(x)}{d x^{6}}-\frac{G_{o} c}{\eta} \frac{d^{4} \sigma_{o}(x)}{d x^{4}}+\frac{E_{o} b}{\eta} \frac{d^{2} \sigma_{o}(x)}{d x^{2}} \\
& -\frac{G_{o} E_{o}\left(b c-a^{2}\right)}{\eta} \sigma_{o}(x)=0,
\end{aligned}
$$

And

$$
\begin{aligned}
& \frac{d^{6} \tau_{o}(x)}{d x^{6}}-\frac{G_{o} c}{\eta} \frac{d^{4} \tau_{o}(x)}{d x^{4}}+\frac{E_{o} b}{\eta} \frac{d^{2} \tau_{o}(x)}{d x^{2}} \\
& -\frac{G_{o} E_{o}\left(b c-a^{2}\right)}{\eta} \tau_{o}(x)=0 .
\end{aligned}
$$

$a$ and $b$ are defined as follows

$a=\frac{1}{2}\left(\frac{t_{1}}{D_{1}}-\frac{t_{2}}{D_{2}}\right), \quad b=\frac{1}{D_{1}}+\frac{1}{D_{2}}, \quad c=\frac{1}{3}\left(\frac{t_{1}^{2}}{D_{1}}+\frac{t_{2}^{2}}{D_{2}}\right)$.

$D_{i}$ is the composite bending stiffness per unit width, which is defined as $D_{i}=E_{i} t_{i}^{3} / 12$.

Solving (14) and (15) is a relatively lengthy and complex and here the solution process is omitted. The reader can find the detailed derivation process in reference [2] and [8]. Here it is worth mentioning that though the interfacial stresses appear decoupled in (14) and (15), $\sigma_{o}(x)$ and $\tau_{o}(x)$ are actually coupled $[2,8]$. The curvatures of the film and substrate layers are found as follows

$$
\begin{aligned}
& \kappa_{f}=-\frac{1}{D_{1}} \int_{-L}^{x} \int_{-L}^{x} \sigma_{o}(x) d x d x+\frac{t_{1}}{2 D_{1}} \int_{-L}^{x} \tau_{o}(x) d x, \\
& \kappa_{s}=\frac{1}{D_{2}} \int_{-L}^{x} \int_{-L}^{x} \sigma_{o}(x) d x d x+\frac{t_{2}}{2 D_{2}} \int_{-L}^{x} \tau_{o}(x) d x .
\end{aligned}
$$

In general, with the interfacial slip, $\kappa_{f} \neq \kappa_{s}$ In the case of ideal interface in (13), $\kappa_{f}=\kappa_{s}=\kappa_{T}$.

\section{RESULTS AND DISCUSSION}

Here the film is Germanium with $E_{1}=105.08 \mathrm{GPa}, v_{1}=0.26$ and $a_{f}=0.56574 \mathrm{~nm}$; the substrate is Silicon with $E_{2}=150$ $\mathrm{GPa}, v_{2}=0.17$ and $a_{s}=0.54306 \mathrm{~nm}[12] . \varepsilon_{m} \approx \bullet 4 \%$. The interface layer is assumed isotropic, so $G_{o}=E_{o} / 2\left(1+v_{o}\right)$

[2]. $v_{o}$ is the Poisson's ratio of the interface layer and assumed $v_{o}=0.2$. Here the relatively compliant interface layer parameters are taken; two cases of $E_{o} / \eta=2 \times 10^{16}$ $\mathrm{Pa} / \mathrm{m}$ and $2 \times 10^{17} \mathrm{~Pa} / \mathrm{m}$ are studied comparatively. The curvatures of the film and substrate of the S-L model are calculated from (17) and (18). The curvatures of the Timoshenko model for the film and substrate are the same. As indicated in (13), the curvature of the Timoshenko model is 
fixed when the dimensions are fixed. In Fig. 2, the following dimensions are used: $t_{1}=t_{2}=2 \mu \mathrm{m}$ and $\mathrm{L}=10 \mu \mathrm{m}$. The uniform curvature of the Timoshenko model is calculated as $\kappa_{T}=14914.1 \mathrm{~m}^{-1}$.

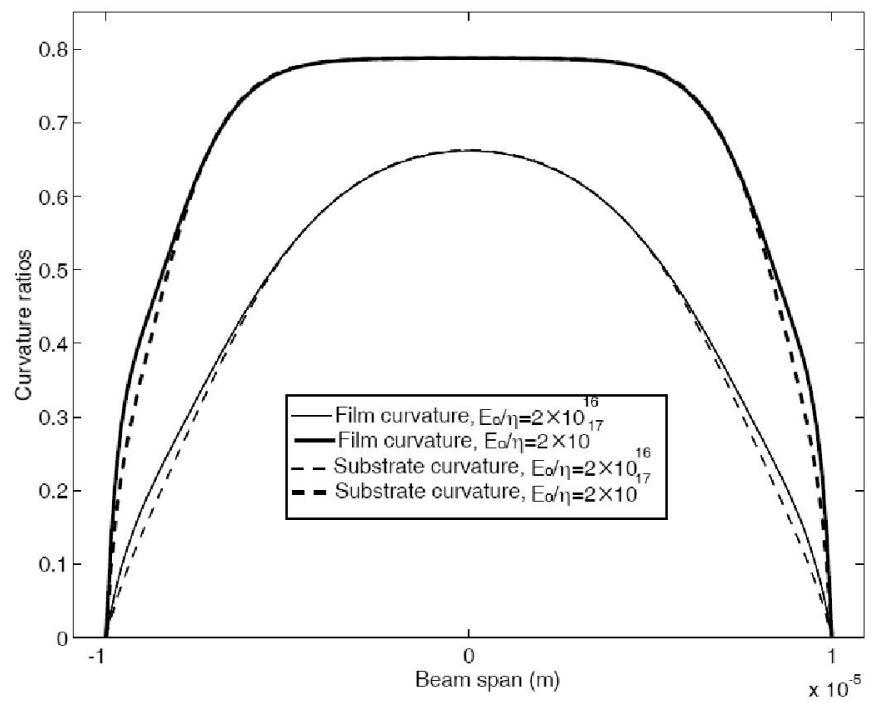

Fig. 2. The curvatures of film and substrate of the S-L model is divided by $\kappa_{T}$. Eo/ $/ \eta$ is taken as $E_{0} / \eta=2 \times 10^{16} \mathrm{~Pa} / \mathrm{m}$ and $2 \times 10^{17} \mathrm{~Pa} / \mathrm{m}$, respectively. The thickness of film layer is $t_{1}=2 \mu \mathrm{m}$ and the thickness of substrate layer is $t_{2}=2 \mu \mathrm{m}, \mathrm{L}=10 \mu \mathrm{m}$ and $\kappa_{T}=14914.1 \mathrm{~m}^{-1}$

As both the theoretical analysis and experiments show that the layers dimensions also have significant influence on the interfacial stresses $\left(\sigma_{o}\right)$ and $\left(\tau_{o}\right)$ as those interfacial parameters (i.e. $E_{o}, G_{o}$ and $\eta$ ) $[1,2]$, so the thickness of both the film and substrate is changed in Fig. 3 to show the effect of layer dimensions. In Fig. $3, t_{1}=1 \mu \mathrm{m}, t_{2}=3 \mu \mathrm{m}$ and $\mathrm{L}=10 \mu \mathrm{m}$. For these dimensions, $\kappa_{T}=9942 \mathrm{~m}^{-1}$ and the curvatures are also divided by these new $\kappa$ T. In Fig. 3 , the curvature difference of the film and substrate becomes significant around the free edges. The film curvatures of both $E_{o} / \eta=2 \times 10^{16} \mathrm{~Pa} / \mathrm{m}$ and $2 \times 10^{17}$ in Figs. 3 experience much more dramatic variation around the free edges.

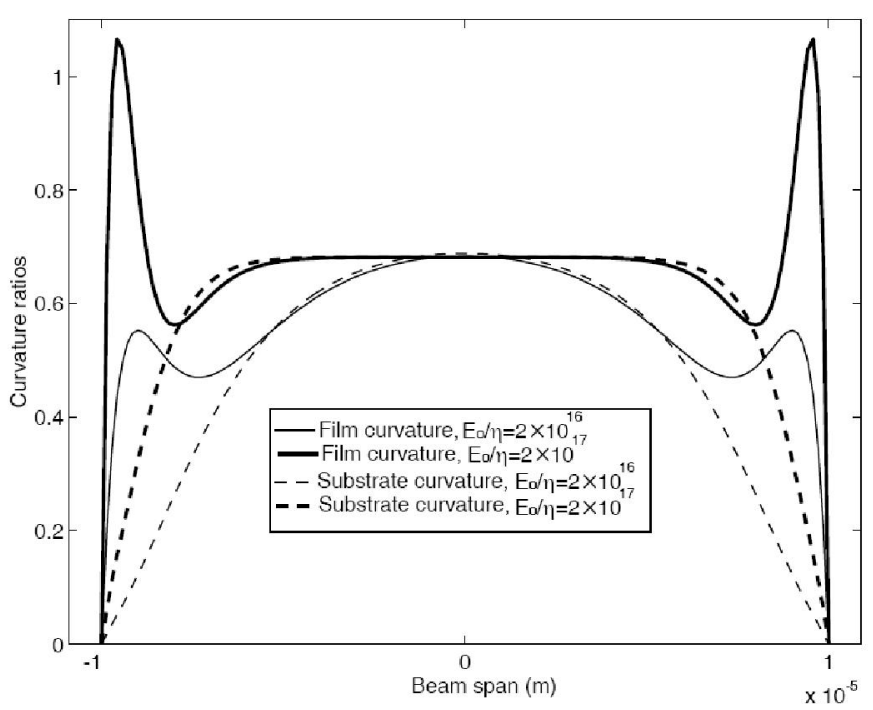

FIG. 3. The curvatures of film and substrate of the S-L model divided by $\kappa_{T} . E_{o} / \eta=2 \times 10^{16} \mathrm{~Pa} / \mathrm{m}$ and $2 \times 10^{17} \mathrm{~Pa} / \mathrm{m} . t_{1}=1 \mu \mathrm{m}, t_{2}=3$ $\mu \mathrm{m}, \mathrm{L}=10 \mu \mathrm{m}, \kappa_{T}=9942 \mathrm{~m}^{-1}$.

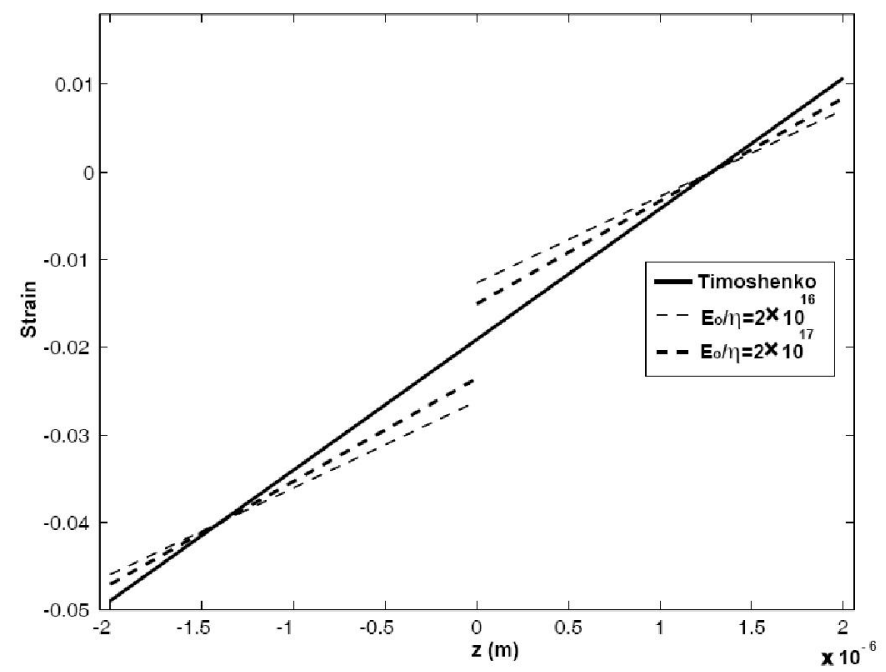

Fig. 4. The comparison of strains calculated by the Timoshenko model and S-L model for different Eo/ $/ \mathrm{s}$. Here $t_{1}=t_{2}=2 \mu \mathrm{m}$ and $\mathrm{L}=10 \mu \mathrm{m}$. The coordinate starts from the interface.

Fig. 4 examines the strains of the film and substrate across the thickness. Clearly for the film/substrate composite with rigid interface (Timoshsenko model), the strain is continuous. While, the strain in those with non-ideal interfaces at the interface is discontinuous. Clearly, as seen from Fig. 4, for larger $E_{o} / \eta$, the strain discontinuity is less. When $E_{o} / \eta$ approaches infinity, the interface is rigid and the discontinuity vanishes. The analysis presented is an analysis on the pure bending of a composite. The interfacial slip results in the discontinuity of the strains inside both the film and substrate. Therefore, the bending moment due to the strain distribution is 
different from that of ideal/rigid interface, which finally leads to the different curvatures.

\section{CONCLUDING REMARKS}

The curvature of non-ideal interface varies with the structure length and differs from that of ideal interface. Therefore, the evaluation of stress state inside the structure based on the measured curvature and the model of ideal interface can result in serious error without properly evaluating the interface state. $E_{o} / \eta$ and $G_{o} / \eta$ are the fitting parameters in the S-L model to be varied to fit the experimental data. Once, the proper $E_{o} / \eta$ and $G_{o} / \eta$ are chosen, (17) and (18) extend the Stoney formula to the application of the composite with interfacial slip.

\section{REFERENCES}

[1] I. C. Noyan, C. E. Murray, J. S. Chey and C. C. "Goldsmith, Finite size effects in stress analysis of interconnect structures," Appl. Phys. Lett., vol. 85, pp. 724-726, 2004.

[2] C. E. Murray and I. C. Noyan, "Finite size effects in thin-film composites," Philos. Mag. A., vol. 83, pp. 3087-3117, 2002.

[3] S. S. Wang and I. Choi, "Boundary-layer effects in composite laminates," J. Appl. Mech., vol. 49, pp. 541-548, 1982.

[4] R. C. Cammarata, K. Sieradzki and F. Spaepen, "Simple model for interface stresses with application to misfit dislocation generation in epitaxial thin films," J. Appl. Phys. vol. 87, pp. 1227-1234, 2000.

[5] F. Shi, K. Chang, K. C. Hsieh, L. Guido and B. Hoke, "Interface structure and adhesion of waferbonded $\mathrm{GaN} / \mathrm{GaN}$ and $\mathrm{GaN} / \mathrm{AlGaN}$ semiconductors," $J$. Appl. Phys., vol. 95, pp. 909-912, 2004.

[6] S. M. Hu, "Film-edge-induced stress in substrates," J. Appl. Phys., vol. 50, pp. 4661-4666, 1979.

[7] S. M. Hu, "Stress-related problems in silicon technology," J. Appl. Phys. 70, R53-R80 (1991).

[8] W. T. Chen and C. W. Nelson, "Thermal stress in bonded joints," IBMJ. Res. Develop., vol. 23, pp. 179-188, 1979.

[9] G. G. Stoney, "The tension of metallic films deposited by electrolysis,"

Proc. R. Soc. Lond. A., vol. 82, pp. 172-175, 1909.

[10] Y. Zhang, Q. Ren and Y. Zhao, "Modelling analysis of surface stress on a rectangular cantilever beam," J. Phys. D: Appl. Phys., vol. 37, pp. 2140$2145,2004$.

[11] L .B. Freund, J. A. Floro and E. Chason, "Extensions of te Stoney formula for substrate curvature to configurations with thin substrates or large deformation," Appl. Phys. Lett., vol. 74, pp. 87-1989, 1999.

[12] L. B. Freund and S. Suresh S, Thin Film Materials: Stress, Defect Formation and Surface Evolution, Cambridge: Cambridge University Press, UK, 2003.

[13]S. Timoshenko, "Analysis of bi-metal thermostats," J. Opt. Soc. Am., vol. 11, pp. 233-255, 1925.

[14] I. A. Blech, I. Blech and M. Finot, "Determination of thin-film stresses on round substrate," J. Appl. Phys., vol. 97, pp. 113525, 2005.

[15] Y. Huang and A. J. Rosakis, "Extension of Stoney's formula to nonuniform temperature distributions in thin film/substrate systems. The case of radial symmetry," J. Mech. Phys. Solids, vol. 53, pp. 2483-2500, 2005.

[16] S. Huang and X. Thang, "Extension of the Stoney formula for filmsubstrate systems with gradient stress for MEMS applications," $J$.

Micromech. Microeng., vol. 16, pp. 382-389, 2006.

[17] Y. Zhang and Y. Zhao, "Applicability range of Stoney's formula and modified formulas for a film/substrate bilayer," J. Appl. Phys., vol. 99, pp. $053513,2006$.

[18] F. Yang and J. C. M. Li, "Diffusion-induced beam bending in hydrogen sensors," J. Appl. Phys., vol. 93, pp. 9304-9309, 2003.

[19] Y. Y. Hu and W. M. Huang, "Elastic and elastic-plastic analysis of multilayer thin films: close-form solutions," J. Appl. Phys., vol. 96, pp. 41544160, 2004. 